\title{
Flexible tag microlab development: Gas sensors integration in RFID flexible tags for food logistic
}

\author{
Estefania Abad ${ }^{\mathrm{a}, *}$, Stefano Zampolli $^{\mathrm{b}}$, Santiago Marco ${ }^{\mathrm{c}}$, Andrea Scorzoni ${ }^{\mathrm{d}}$, Barbara Mazzolai ${ }^{\mathrm{e}}$, \\ Aritz Juarros ${ }^{\mathrm{a}}$, David Gómez ${ }^{\mathrm{a}}$, Ivan Elmi ${ }^{\mathrm{b}}$, Gian Carlo Cardinali ${ }^{\mathrm{b}}$, José M. Gómez ${ }^{\mathrm{c}}$, \\ Francisco Palacio ${ }^{c}$, Michelle Cicioni ${ }^{d}$, Alessio Mondini ${ }^{\mathrm{e}}$, Thomas Becker ${ }^{\mathrm{f}}$, Ilker Sayhan ${ }^{\mathrm{f}}$ \\ a Micro and Nanotechnology Department, Fundación Tekniker, Eibar, Spain \\ b CNR-IMM Sezione di Bologna, Italy \\ c Universitat de Barcelona, Spain \\ ${ }^{\mathrm{d}}$ DIEI, University Perugia, Italy \\ e SSSA CRIM lab, Pisa, Italy \\ ${ }^{\mathrm{f}}$ EADS Deutschland GmbH, Corporate Research Centre, München, Germany
}

Available online 5 July 2007

\begin{abstract}
The enabling technologies for the development of a flexible tag microlab for food monitoring during the logistic chain will be presented. The realisation of the system includes the integration of physical and chemical sensors with Radio Frequency IDentification (RFID) communication capabilities. The first ISO 15693 compliant semi-active tag prototype, including low power control electronics, RFID antenna, commercial sensors, memory and a thin film battery, is shown together with the development of novel ultra-low power hotplates required for this application and the process, based on the use of anisotropic conductive adhesive (ACA) flip chip technology, for gas sensors integration onto flexible substrates.
\end{abstract}

(C) 2007 Elsevier B.V. All rights reserved.

Keywords: Flexible substrate technologies; Gas sensors hotplates; RFID communication; ACA flip chip; ISO 15693

\section{Introduction}

In the framework of the "GoodFood" FP6 Integrated Project (IP) [1], a flexible tag microlab (FTM), integrating physical and chemical sensing and Radio Frequency IDentification (RFID) communication capabilities, for food monitoring during the logistic chain has been proposed (see Fig. 1). The idea for this device is a flexible label, hosting different sensor technologies and a RFID interface for wireless data exchange, capable of monitoring the quality of food during transport, storage and vending.

Nowadays RFID flexible labels are coming into widespread use. These systems are simple wireless transponders with integrated memory chips. First attempts have been made to integrate temperature sensors onto the same substrate [2]. However, at the moment there are no commercial labels including chemical sensors. In this paper, we present the integration of gas sensors onto

\footnotetext{
* Corresponding author. Tel.: +34 9432067 44; fax: +34 943202757.

E-mail address: eabad@tekniker.es (E. Abad).
}

flexible substrates together with an RFID transponder to give an innovative system as required for the application.

RFID technology in the $13.56 \mathrm{MHz}$ band was chosen since it is the best compromise for integration on a flexible tag. Furthermore, this band is very suitable for the food logistic application, considering possible constraints, such us the surrounding environment (e.g. humidity) and range of communication. In order to be compliant with recent RFID developments, the ISO 15693 standard has been selected.

This visionary application involves both the fabrication of the so-called inlay, which is the flexible substrate with all components needed for the FTM assembled on it, and the development of new ultra-low power consumption substrates for gas sensor integration. Particular assembly and packaging issues for these critical components are also addressed.

\section{RFID flexible tag}

Flexible substrate and component assembly technologies [3] for the FTM have been developed and/or optimised. A simple process for the fabrication of flexible substrates has been 


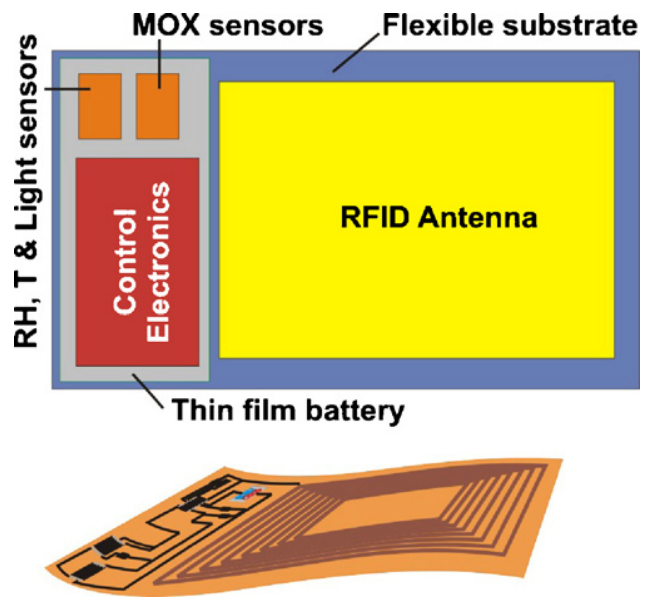

Fig. 1. Main functional blocks of the FTM for food logistics.

implemented. The outline of this process is presented in Fig. 2. The material employed is the DuPont ${ }^{\mathrm{TM}}$ Pyralux ${ }^{\circledR}$ AP $8525 \mathrm{R}$ double-sided, copper-clad laminate (Kapton) [4], which is an adhesiveless laminate for flexible printed circuit applications. The Kapton has a thickness of $50 \mu \mathrm{m}$ and the copper layer has a thickness of $18 \mu \mathrm{m}$ on each side.

In this procedure, the vias definition in Kapton was performed directly by femtosecond laser ablation. Then, the copper interconnections of the two metal levels necessary for the interconnect were generated by standard photolithography and wet etching. Finally, contacting through the vias was also implemented. Further details of this procedure are given elsewhere [5]. An example of the double-sided flexible circuit (a) and antenna (b) fabricated using this process is presented in Fig. 3.

The flexible antenna of the tag is composed of a five-turn spiral-shaped inductor (track thickness of $18 \mu \mathrm{m}$, track width of

Pyralux AP 8525R: Kapton foil $(50 \mu \mathrm{m})$ with $\mathrm{Cu}(18 \mu \mathrm{m})$ double-sided

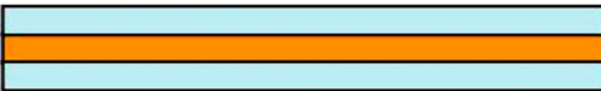

Step 1: Open vias and windows in kapton and $\mathrm{Cu}$

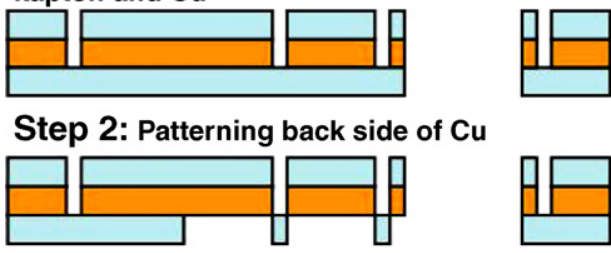

Step 3: Patterning front side of $\mathrm{Cu}$

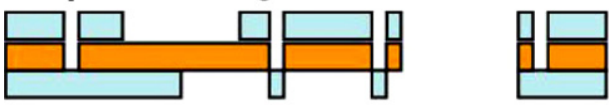

Step 4: Filling the vias to make the electrical contact
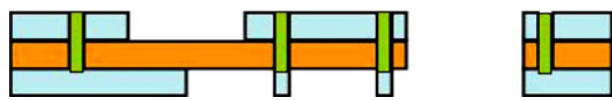

Fig. 2. Process design for flexible substrates fabrication.

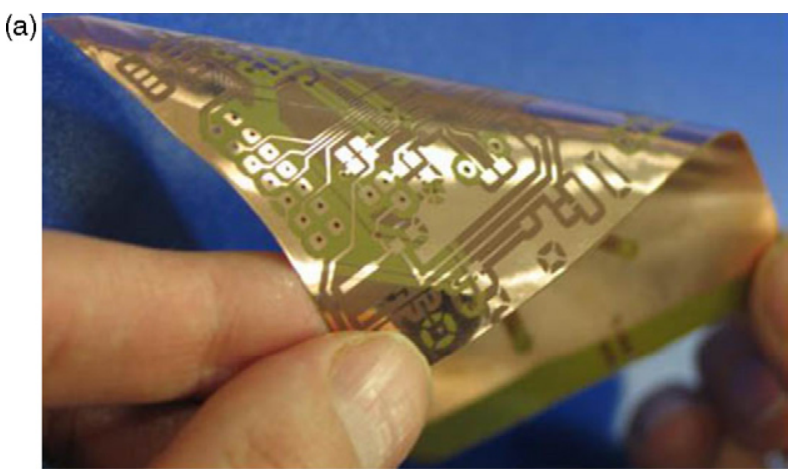

(b)

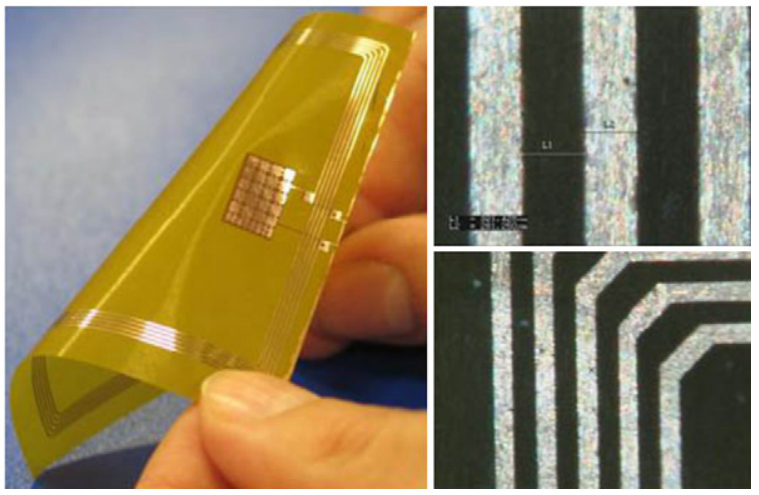

Fig. 3. Photographs and microscope images of the flexible circuit (a) and the flexible antenna (b). Details of the five copper tracks of the inductor

$300 \mu \mathrm{m}$ and spacing between two tracks of $300 \mu \mathrm{m}$, as shown in the microscope images of Fig. 3(b)) and a parallel trimmable plate capacitor to tune the frequency to $13.56 \mathrm{MHz}$.

The flexible tag was chosen to be compliant with the ISO 15693 RFID standard. The implemented system is a semi-active tag with a passive read-out with a reading distance of about $15 \mathrm{~cm}$ and a battery powered sensing part. Fig. 4 shows the first prototype of the developed FTM. For this prototype, packaged chips were integrated on the flexible circuit using conventional assembly technologies.

The main functional blocks include a microcontroller for sensor control and signal acquisition, a RFID front-end and a complex programmable logic device (CPLD) for signal modulation/demodulation, commercial sensors (relative humidity, temperature and light), an EEPROM memory and a thin film flexible battery. Regarding sensing capabilities, this prototype contains a Sensirion SHT15 relative humidity and temperature sensor and a commercial light sensor (SLDC-61N1 + OPA349 amplifier), while ultra-low power MOX sensor integration (see Section 3) will be incorporated in the final version. The low power electronics are based on a Texas Instruments MSP430 microcontroller [6]. This microcontroller has been programmed to manage both the data acquisition and the RFID communication. The acquisition is achieved by two methods: alarm-triggered and time-triggered. The value of time acquisition and the alarms range is defined during the initialization of the tag. If an alarm is detected, the microcontroller stores the value of each sensor and the alarm time in the memory. 


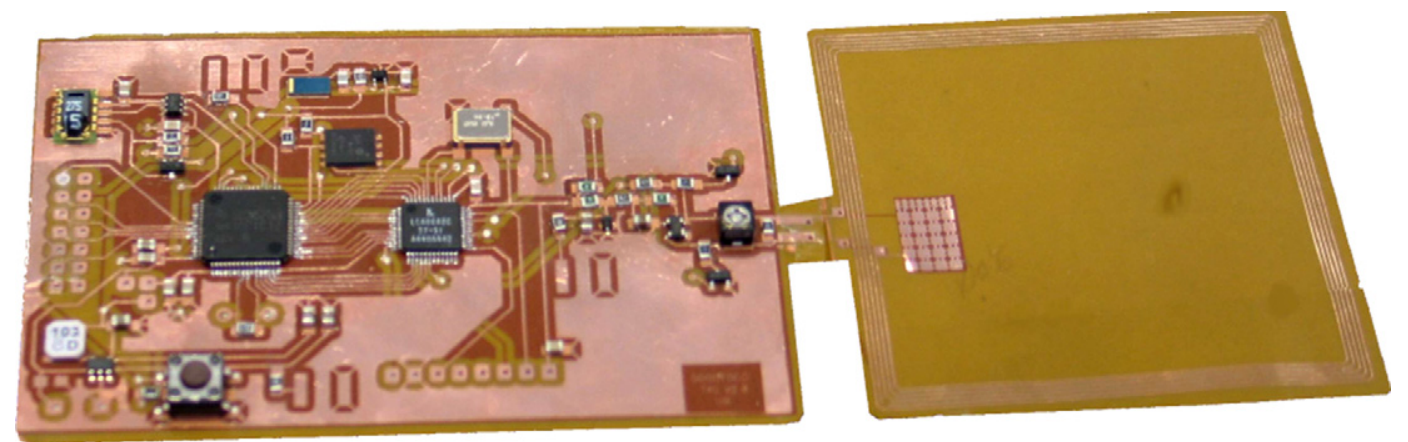

Fig. 4. FTM first prototype including relative humidity, temperature and light sensors and a flexible antenna. A credit card size was chosen for the tag.

\section{Ultra-low power gas sensors integration}

Ultra-low power consumption hotplates (ULPHP) for MOX sensor integration on flexible substrates have been developed [7]. The hotplates were designed and optimized to fulfil the requirements of the RFID flexible tag application: ultra-low power consumption due to low energy availability, reduced die dimensions for tag flexibility and a die design compatible with reliable encapsulation on flexible substrates.

Front-side bulk micromachined suspended dielectric membranes were designed and fabricated, with a single $\mathrm{Pt}$ metallization layer implementing both the heating resistor and the sensing layer contacts on the same level. A circular hotplate layout was chosen in order to optimize temperature uniformity on the hot-spot of the suspended structure. Several devices were designed with different hotplate diameters ranging from 50 to $100 \mu \mathrm{m}$. With an $80 \mu \mathrm{m}$ circular membrane, only $8 \mathrm{~mW}$ are necessary to reach $400^{\circ} \mathrm{C}$ operating temperature. Fig. 5 shows a SEM image of a $1.5 \mathrm{~mm} \times 1.0 \mathrm{~mm}$ die implementing a 4 -sensor array.

The integration of MOX sensors hotplates requires specific assembling methods and protection of the chips from the environment. Flip chip technology utilizing anisotropic conductive adhesives (ACAs) has been proved to be a possible solution for MEMS packaging [8,9]. Using this technology, a special procedure has been designed for the integration of the ULPHP, involving the following main steps (see Fig. 6):

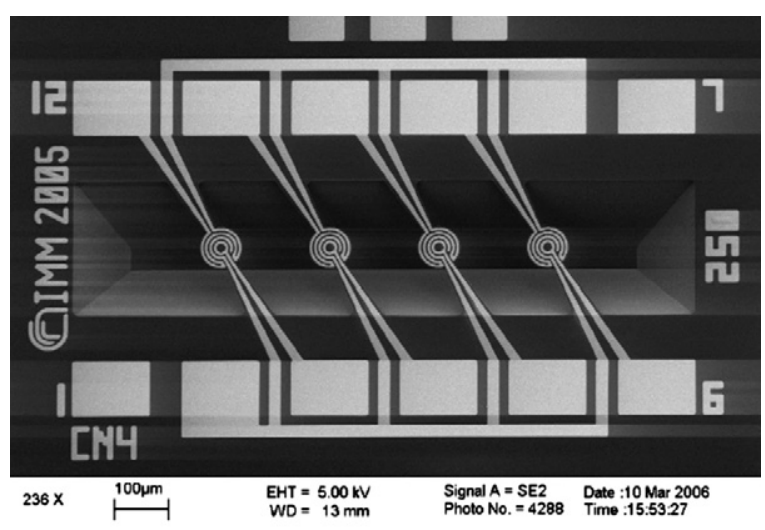

Fig. 5. SEM image of the ultra-low power hotplates.
(1) Window opening by femtosecond laser ablation.

(2) Patterning of the electrical contacts.

(3) ACAs flip chip for assembly.

(4) Polymer casting and curing for encapsulation.

A simple flexible substrate layout was designed in order to prove the viability of ACA flip chip assembly of the ULPHP. For this initial trial, the single-sided flexible substrates shown in Fig. 7 were produced. Each test substrate includes a $1.15 \mathrm{~mm} \times 0.4 \mathrm{~mm}$ laser-ablated window and 12 copper pads (140 $\mu \mathrm{m} \times 100 \mu \mathrm{m}$ size and $60 \mu \mathrm{m}$ spaced) linked to connectors. The ACA bonding can be tested by measuring the resistance at the corresponding connectors with a multimeter.

The anisotropic conductive adhesives can provide unidirectional conductivity, which is always in the vertical, or $Z$-axis. The directional conductivity is achieved by using a relative low volume loading of conductive filler. The low volume loading,

\section{Pyralux AP 8525R: Kapton foil $(50 \mu \mathrm{m})$ with $\mathrm{Cu}(18 \mu \mathrm{m})$ double-sided}

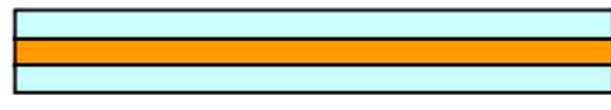

Step 1: Windows opening by femtosecond laser ablation

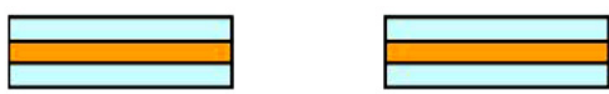

Step 2: Patterning of electrical contacts

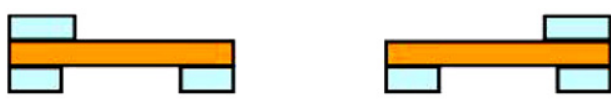

Step 3: ACA flip chip bonding

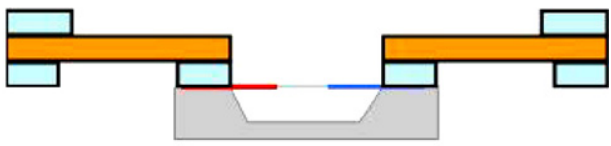

Step 4: Polymer casting and curing

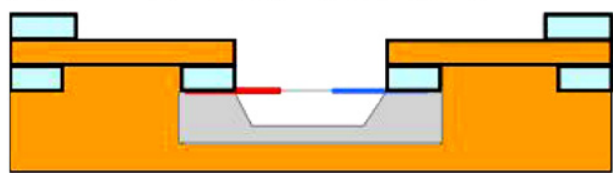

Fig. 6. Process design for the MOX sensor integration. 


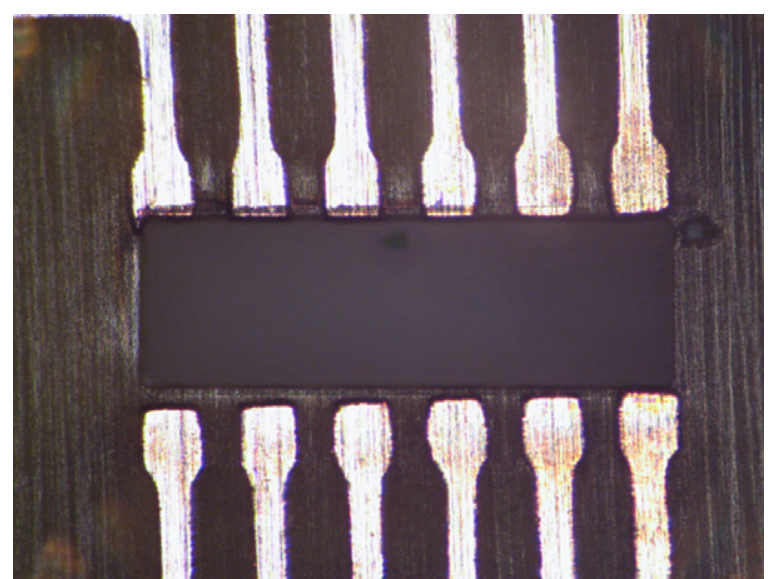

Fig. 7. Flexible substrate fabricated to prove the viability of ACA flip chip assembly of ULPHP.

which is insufficient for interparticle contact, prevents conductivity in the plane of the adhesive. The $Z$-axis adhesive is placed between the surfaces to be connected and pressure and/or heat is applied to form the bond, as illustrated in Fig. 8(a). This type of product is now being used in flexible circuit interconnection, especially in copper/polyimide circuits. Due to their anisotropic conductivity, these adhesives can be deposited over the entire surface, thus facilitating the material application and avoiding the use of a dielectric layer and the formation of bumps onto the chip pads.

(a)

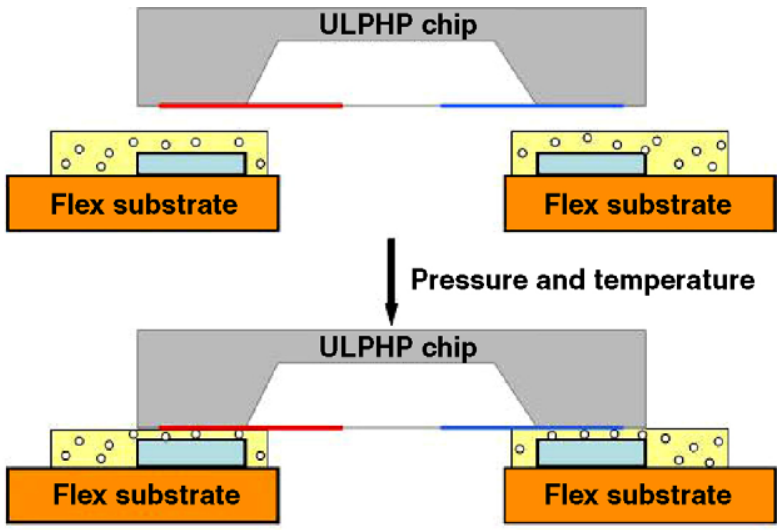

(b)

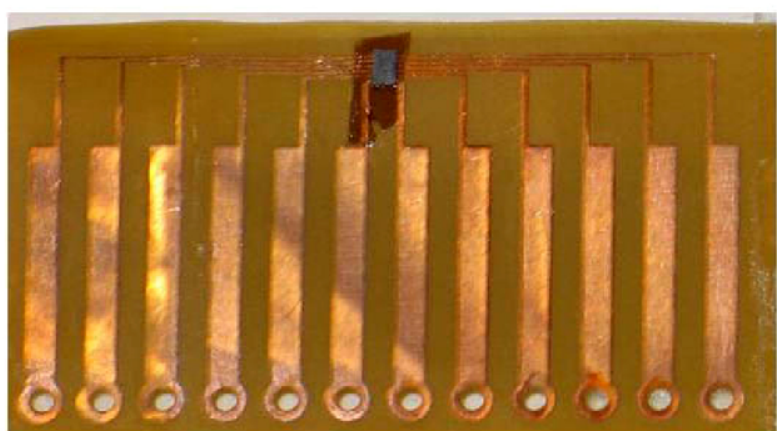

Fig. 8. (a) Schematic view of the thermo-compression bonding using ACAs. Conductive particles are trapped between the flexible circuit and the chip pads. (b) ULPHP chip assembled on the flexible substrate.
Table 1

Pre-tacking and bonding parameter for the ACA 5460R from $3 \mathrm{M}^{\mathrm{TM}}$

\begin{tabular}{lclr}
\hline Step & Temperature $\left({ }^{\circ} \mathrm{C}\right)$ & Pressure $(\mathrm{MPa})$ & Time $(\mathrm{s})$ \\
\hline Tacking & 80 & $0.1-1$ & 5 \\
Bonding & 170 & 3 & 20 \\
\hline
\end{tabular}

The adhesive employed to assemble the ULPHP onto the flexible substrate was the $Z$-axis film $5460 \mathrm{R}$ from $3 \mathrm{M}^{\mathrm{TM}}$. The $5460 \mathrm{R}$ ACA film is a $40 \mu \mathrm{m}$ cyanate ester and epoxy/thermoplastic blend loaded with $7 \mu \mathrm{m}$ size gold-plated nickel particles. The film is attached to a liner to facilitate the handling and supports a maximum current $100 \mathrm{~mA} / 0.1 \mathrm{~mm}^{2}$. Using this film it should be possible to achieve an interconnect resistance of less than $0.05 \Omega$.

Electrical interconnections in ACA flip chip bonding are formed by a thermo-compression cycle. The procedure for the flip chip assembly includes several steps: (1) heat pre-tacking the film to the flexible circuit, (2) removal of the release liner, (3) ULPHP flipping and alignment to the substrate and (4) bonding by a thermo-compression cycle. All these operations are performed in a Dr. Treski AG 8800 flip chip station with alignment errors below $10 \mu \mathrm{m}$. Manufacturer's recommendations were used for the pre-tacking and thermo-compression bonding steps. The values employed for these steps are gathered in Table 1. The heating was realised by placing the test flexible substrate on the hot chuck of the flip chip system. The temperature control over the film and the applied pressure are the key parameters to achieve a good bonding. For this reason, a thermocouple was employed during the thermo-compression cycle to assure that the required film temperature was reached. The pressure was applied by pushing the ULPHP towards the substrate using the vacuum gripper of the system with a force previously calibrated.

Fig. 8(b) shows an image of an ULPHP chip assembled on the flexible substrate using the $Z$-axis film 5460R following the procedure described previously. Electrical and mechanical tests confirm that the bond is very reliable.

The electrical behavior of the bonding connections was characterized using some preliminary ULPHP test dies integrating six heater resistors. At first, the series resistance formed by the heaters and the ACA bonding connections was compared to the resistance of the heaters of a new, not encapsulated die. The resistance was measured with an $I-V$ ramp in the range from $-10 \mathrm{~mA}$ to $+10 \mathrm{~mA}$, which is higher than the $7 \mathrm{~mA}$ maximum current expected during the ULPHP sensor operation. The comparison of the average curves of six heaters is shown in Fig. 9, and no significant difference can be discerned between the resistance of the stand-alone ULPHP and the ULPHP integrated on the flexible substrate. The characteristic non-linearity of the $I-V$ curve is due to the temperature increase of the hotplate heater resistors while dissipating power.

To evaluate the short-term stability of the electrical connections, a $7 \mathrm{~mA}$ current was applied continuously for $15 \mathrm{~h}$ and the heater resistance was acquired. As can be seen from Fig. 10, the heater resistance of $123.1 \Omega$ does not change during the $15 \mathrm{~h}$ of operation. 


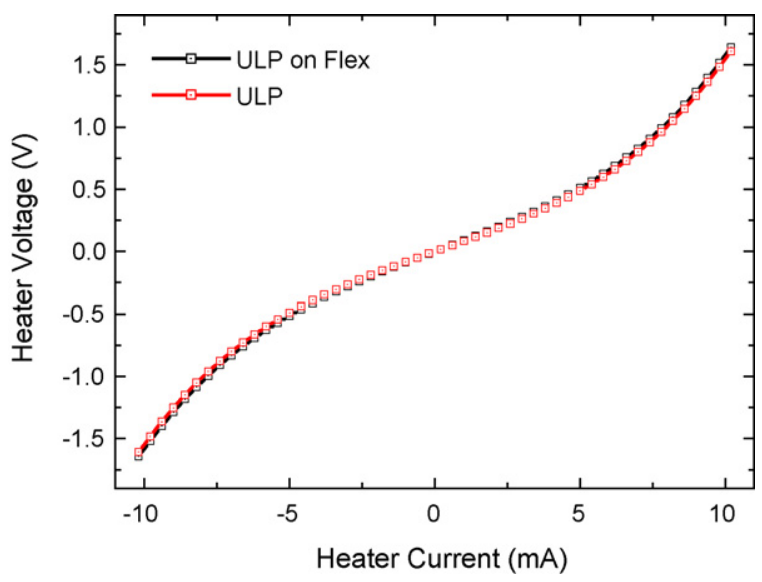

Fig. 9. Comparison of resistance measurements between ULPHP (red) and encapsulated ULPHP (black). Each curve is the average of six different heaters within the test die. (For interpretation of the references to color in this figure legend, the reader is referred to the web version of the article.)

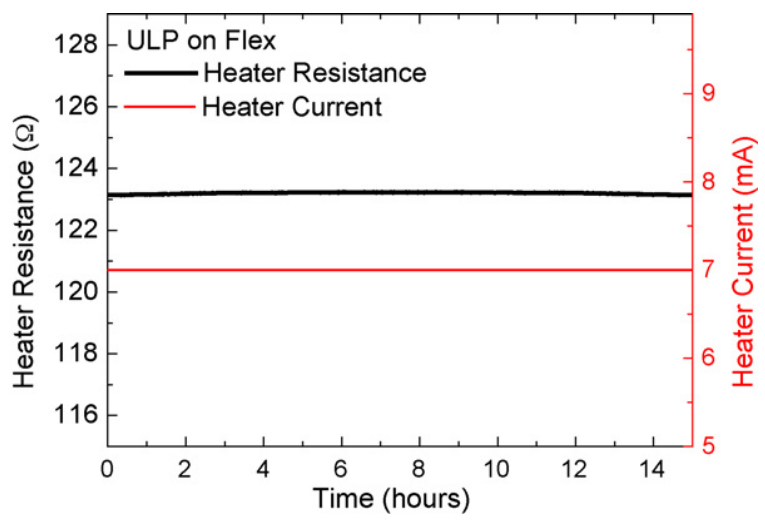

Fig. 10. Electrical short-term stability of the ACA flip chip bonding. The heater resistance does not change significantly during $15 \mathrm{~h}$ of hotplate operation at $7 \mathrm{~mA}$.

The above measurements experimentally confirm that the $7 \mathrm{~mA}$ current supply necessary for powering the ULP hotplates and the pad dimensions of $0.012 \mathrm{~mm}^{2}$ are compatible with the specifications of the ACA film, resulting in approximately half of the maximum specified current density of $100 \mathrm{~mA} / 0.1 \mathrm{~mm}^{2}$.

\section{Conclusions}

Flexible substrate technology and assembly of components, in particular of the new ULPHP specifically developed for MOX sensor integration using ACAs flip chip technology, have been accomplished with the aim of developing a FTM inlay for food logistic control. The first prototype of the ISO 15693 compliant semi-active tag, including low power control electronics, RFID antenna, commercial sensors, memory and a thin film battery, has been presented. Ultra-low power consumption MOX sensors hot plates have been developed to fulfil the requirements of the RFID flexible tag application. Finally, the assembly of the ULPHP on flexible substrates using ACAs flip chip technology has also been proved. Considering that the HP is the mechanically critical structural component of the gas sensors, these achievements will allow the integration of chemical sen- sors in the RFID tag that represents the major innovation of this FTM realisation.

\section{Acknowledgements}

This work is being financed by the EU Frame Program VI within the IST priority. The authors wish to thank Mr. Javier Berganzo and Mr. Rafael Salido for the technical support with the flip chip station. The authors also wish to acknowledge $3 \mathrm{M}$ for providing ACA samples.

\section{References}

[1] http://www.goodfood-project.org/.

[2] K. Opasjumruskit, T. Thanthipwan, O. Sathusen, P. Sirinamarattana, P. Gadmanee, E. Pootarapan, N. Wongkomet, A. Thanachayanont, M. Thamsirianunt, Self-powered wireless temperature sensors exploit RFID technology, IEEE Pervasive Comput. 5 (2006) 1268-1536.

[3] D. Numakura, Flexible circuits, in: Printed Circuits Handbook, fifth ed., McGraw Hill, New York, 2001, p. 1205.

[4] http://www.dupont.com/fcm/products/pyralux.html/.

[5] E. Abad, V.S. Raffa, B. Mazzolai, S. Marco, A. Krenkow, T. Becker, Development of a flexible tag microlab, Proc. SPIE 5836 (2005) 599-606.

[6] MSP430x1xx Family user's Guide, Texas Instruments (SLAU049E).

[7] S. Zampolli, I. Elmi, E. Cozzani, M. Passini, G.C. Cardinalli, M. Severi, Ultra-low-power MOX sensors arrays for wireless sensor networks and RFID applications, EUROSENSORS XX Book of Abstracts, vol. 2, 2006, pp. 292-293.

[8] R.S. Pai, K.M. Walsh, The viability of anisotropic conductive film as a flip chip interconnect technology for MEMS devices, J. Micromech. Microeng. 15 (2005) 1131-1139.

[9] A. Johansson, J. Janting, P. Schultz, K. Hoppe, I.N. Hansen, A. Boisen, SU8 cantilever chip interconnection, J. Micromech. Microeng. 16 (2006) 314-319.

\section{Biographies}

Estefania Abad received a degree in physic sciences in 1995 from the University of Cantabria. In 2001, she obtained her PhD in physics at the Physics Department of the University of Oviedo, working on magnetic interactions in intermetallic compounds. During 2000 and 2001, she combined this activity with a position as assistant professor in the University of Oviedo. Then, she joined TEKNIKER, that sponsored her to get a MSc degree in microsystems and nanotechnology at Cranfield University in 2002. Since October 2002, she has been a researcher of the Micro and Nanotechnology Department at Tekniker working on MEMS fabrication and polymer replication technologies. Her research interest also covers flexible circuit technologies.

Stefano Zampolli graduated in physics at the University of Bologna in 2000, discussing a thesis on the application of Fuzzy Logic for detection of buried landmines. Grant student at CNR-IMM Bologna from 2000 to 2004, since 2005, he has been a researcher at CNR-IMM Bologna working primarily on gas sensor design and characterization and data processing within the sensor and microsystem R\&D program.

Santiago Marco is associate professor (Profesor Titular) at the Departamento d'Electronica of Universitat de Barcelona since 1995. He received the degree in physics from the Universitat de Barcelona in 1988. From 1989 to 1990, he was working in the electro-optical characterization of deep levels in GaAs. From 1990 to 1993, he was regular visitor of the Centro Nacional de Microelectrònica, Bellaterra, Spain. In 1993, he received his PhD (honor award) degree from the Departamento de Física Aplicada i Electrònica, Universitat de Barcelona, for the development of a novel silicon sensor for in vivo measurements of the blood pressure. In 1994, he was a post-doc researcher at the Department of Electronic Engineering, Universita di Roma 'Tor Vergata', working in data processing for artificial olfaction. He has published about 50 papers in scientific journals and 
books, as well as more than 100 conference papers. His current research interests are two-fold: chemical instrumentation based on intelligent signal processing and microsystem modeling.

Andrea Scorzoni received his doctoral degree in electronics in 1989. Since 1983, he has been working under various contracts in Bologna at CNR-IMM (former CNR-LAMEL.) Since 1998, he is professor of electronics at the Department of Electronic and Information Engineering (DIEI), University of Perugia. The areas of interest include embedded digital systems and RFID systems, ohmic contact resistivity measurements and modeling for both VLSI applications and SiC-based integrated circuits, reliability and degradation mechanisms of semiconductor devices with particular focus on electromigration phenomena, solid-state, silicon-based, radiation sensors and gas sensors. He has authored and coauthored more than 60 peer-reviewed technical papers.

Barbara Mazzolai received her Laurea degree in biology (with Honours) from the University of Pisa, Italy, in 1995. From 1994 to 1998, she worked at the Institute of Biophysics of the National Research Council on environmental topics and in particular, on mercury pollution. In 1998, she received her master's degree in eco-management and audit schemes (EMAS) organized by the Scuola Superiore Sant'Anna (SSSA), Pisa, Italy. Then, she had a research assistant position at Center for Research in Microengineering (CRIM) Laboratory of SSSA from February 1999 until June 2004. From July 2004, she obtained a temporary position of assistant professor in bioengineering at SSSA. Her main research interests are in the fields of environmental microrobotics, microsystems and sensors for environmental, agrofood and biomedical applications. She is currently working on several European and international projects focused on these fields.

Aritz Juarros industrial technical engineer by the Bask Country University in 2000 BEng Bachelor of Engineering in Electrical \& Electronics by the North East Wales Institute of Higher Education (NEWI), Wrexham, UK. He studied a master of science in microsystems Engineering in Furtwangen Germany 2003-2004. In 2004, he joined Tekniker Fundation, working in MEMS fabrication technologies and polymer replication.

David Gómez received his degree in physics in 1993 from the Universidad de Cantabria, Spain, where until 1995, he was collaborating in research activities at the Condensed Matter Department. In 2000, he obtained his PhD degree from the Universidad del País Vasco, Spain, related to the molecular dynamics of polymer glasses. He joined the Micro- and Nanotechnology Department of Tekniker in 2001, where he has been involved in cleanroom technologies for sensors development and polymer replication. Since 2003, his work has been also focused on the application of femtosecond phenomena to micromachining applications.

Ivan Elmi graduated in physics at the University of Bologna in 1998, discussing a thesis on characterization of sensors for benzene detection. From April 1999, he worked as a grant student and since 2005, he has been a researcher at CNR-IMM Sez. di Bologna within the sensor and microsystem $R \& D$ program, primarily involved in development of gas sensors and miniaturized gas-chromatographic systems and in microsystem design and fabrication technologies.

Gian Carlo Cardinali received a degree in electronic engineering from the University of Bologna, Italy, in 1979. Since 1982, he has been working at the IMM-CNR Institute. His scientific interests are in the areas of design, fabrication and testing of electronic devices and microsystems. From 1996 onwards, he has been involved in research projects dealing with the implementation of systems for air quality monitoring based on micro-gas sensors. He is also dealing with the development of dedicated microelectronic processes suitable for integration into microsystems.

José M. Gómez is associate professor at the Universitat de Barcelona since 2003. He received his BS degree in Physics from the Universitat de Barcelona in 1994, and his electronic engineer degree in 1996. He finished his $\mathrm{PhD}$ in 2000. Between 1998 and 2003, he has been working for Gas Natural, PriceWaterhouseCoopers and IBM. His current research interest is wireless sensor networks and embedded systems.

Francisco Palacio was born in Barcelona, Spain, on 1971. He received a degree in electronic engineering from University of Barcelona in 2004. In the same year, he joined the ISPlab as a PhD student.

Michelle Cicioni graduated cum laude in electronic engineering at the University of Perugia (Italy) in 2004. He is currently a PhD student at the Department of Electronic and Information Engineering (DIEI) of the University of Perugia, Italy. His research interests are in RFID systems and brushless motor control systems.

Alessio Mondini is a PhD student of microsystems at the University of Torvergata, Roma, Italy. He received his Laurea degree in electronic engineering from the University of Pisa, Italy in 2002. In 2003, he worked in the National Energy Company (ENEL) with a stage program focused on data acquisition system and sensor conditioning for physical measures into the geothermal wells. From May 2004, he has been working at CRIM Laboratory of Scuola Superiore Sant'Anna. His main research interests include microsystems technologies, communication system and miniaturised data acquisition system for sensor networks.

Thomas Becker was born in 1967. He graduated in electrical engineering and microsystems technology at the IMSAS, University of Bremen, Germany in 1995 and he received his doctoral level (Dr.-Ing.) in 2000. In 1994, he joined DaimlerChrysler Research and Technology in Munich (Germany) for microsystems and electronics, which became the EADS Corporate Research Centre in 2000. There, he runs the gas-sensing laboratory within the Chemical Sensors Group. Within this perimeter, he has been working on various aspects of sensor technology, sensor applications, micro reactor systems and polymer technologies. From 2002 until 2004, he was appointed as key technology area manager for microsystems, electronics and microelectronics. In 2004, he was appointed manager for safety and security-related aerospace topics. Moreover, he is guest lecturer for microsystems technology at the private University of Applied Science in Isny/Allgaeu since 2003. He is author and co-author of several scientific papers and text books and holds several patents in the area of microsensors and sensor systems.

Ilker Sayhan, born in 1976, graduated 2003 in physics from the Isny Applied Sciences University. In 2004, he started his PhD at EADS Corporate Research Centre in Germany in the Department of Sensor Systems and Integration as a student of the University of Barcelona. Amongst other things, he is working in the field of chemical sensors for maintenance, safety and security. His research focus is on power saving alternative working principles for metal oxide gas sensors and their system integration. 